\title{
XIX.
}

\section{Ueber einen Fall von Gliom im dorsalen Abschnitt des Pons und der Medulla oblongata.}

\author{
Von \\ F. Jolly. \\ (Hierzu Taf. XIII. und Zinkographien.)
}

Die Erkrankungen der Brückengegend und des verlängerten Marks sind wegen der mannigfachen Faserzüge, Nervenwurzeln und grauen Massen, die hier in nächster Nachbarschaft beisammen liegen und deren Functionsstörung je nach dem Sitz und der Ausdehnung des Krankheitsherdes in charakteristischen Combinationen zu Tage tritt, für die Diagnostik von unversiegbarem Interesse.

So leicht es nun in der Regel ist, die Ausdehnung solcher Läsionen wenigstens in ihren gröberen Verbältnissen schon zu Lebzeiten der Kranken festzustellen, so harren doch noch manche Punkte der Aufklärung und wir sind noch lange nicht so weit, um über die physiologische Bedeutung aller Theile jenes Gebiets und über die Localisation verschiedener wichtiger Symptome abschliessende Ergebnisse zu besitzen. Die ausführliche Mittheilung von anatomisch und klinisch genau untersuchten Fällen muss daher sowohl im physiologischen wie im pathologischen Interesse als wünschenswerth bezeichnet werden. Dabei ist von vornherein daran zu erinnern, dass nicht allein der Sitz einer pathologischen Veränderung, sondern auch ihre Beschaffenheit nnd die Art ihrer Entwicklung für das Auftreten oder Fehlen gewisser Symptome von Bedeutung ist, und dass nur mit Berücksichtigung dieses Umstandes eine befriedigende Deutung scheinbar widersprechender Beobachtungen gegeben werden kann. 
Der Fall, der zu diesen Bemerkungen Anlass giebt, wurde in vivo bereits vor längerer Zeit, als die Krankheitserscheinungen in charakteristischer, eine Localdiagnose ermöglichender Weise ausgebildet waren, von mir in der Gesellschaft der Charitéärzte vorgestellt"). Die mikroskopischen Präparate habe ich sodann vor Kurzem in der Gesellschaft für Psychiatrie und Nervenkrankheiten mit dem Projectionsapparat demonstrirt und bei dieser Gelegenheit einige $\mathrm{Be}$ merkungen über die Localisation einzelner Symptome angeknüpft**). Eine zusammenhängende und eingehendere Besprechung wird trotzdem nicht überflüssig erscheinen.

Luise H., Nähmaschinenarbeiterin, 25 Jahre alt, wurde am 16. Januar 1891 in die Nervenklinik der Charité aufgenommen.

Anamnese. Beide Eltern an der Cholera gestorben. Zwei Brüder lebend gesund. In der Familie keine Nervenkrankheiten vorgekommen.

Patientin war als Kind gesund, hat auch später keine erheblichen Krankheiten durchgemacht. Syphilitische Infection wird geleugnet. Zeichen von Syphilis sind nicht auffindbar. 5 Jahre vor der Aufnahme litt sie 6 Wochen lang an Heiserkeit, was sich später nicht wiederholte.

Weihnachten 1889 erkrankte sie an Influenza, hatte eine Nacht und einen Tag starkes Frostgefühl mit Uөbelkeit und Schwindel, ging aber trotzdem zur Arbeit, erholte sich vollständig.

Am 15. März 1890 bemerkte sie beim Erwachen, nachdem sie Tags vorher Frost und Hitze gehabt hatte, dass ihr Gesicht schief nach rechts verzogen war und dass sie das linke Auge nicht schliessen konnte (linksseitige $\mathrm{F}$ acialislähmung). Im Uebrigen keinerlei Beschwerden, weder Kopfschmerz, noch Sohwindel, noch Störungen in den Extremitäten.

Mitte des Sommers 1891 litt sie acht Tage lang an Schwindel, Uebelkeit und Erbrechen, jedoch ohne Kopfsohmerzen. Einen Tag lang war sie unfähig, feste Speisen zu schlucken, konnte nur mit sehr viel Flüssigkeit einzelne Brocken hinunterwürgen. Dann fühlte sio sich wieder gesund, abgesehen von der Gesichtslähmung, die fortbestand, sich vorübergehend nach poliklinischer elektrischer Bebandlung besserte, dann wieder zunahm, um nach erneutem Elektrisiren abermals etwas besser zu werden.

8 Tage vor Weihnachten 1890 abermals einen Tag lang Unfähigkeit, feste Spoisen zu schlucken. Gleichzeitig starkes Sehwindelgefühl und Erbrechen, was 8 Tage hindurch fortbestand. Dann konnte sie 3-4 Tage lang "schlecht sehen", sah alles wio durch einen Schleier. Von da an häufig Beschwerden beim Schlucken und am 12. Januar 1891 während des Essens ein Erstickungsanfall, der erst aufhörte, als dio im Schlunde steckenden Speisen wieder heransbefördert waren. Seitdem wagte sie auch keine Flüssigkeit mehr zu schlucken.

*) Berliner klin. Wochensohr. 1892. No. 24.

**) Neurologisches Centralbl. 1894. No. 11. 
Status praesens bei der Aufnahme.

Patientin befindet sich in etwas reducirtem Ernährungszustande, bei pöllig freiem Sensorium. Klagt über quälenden Durst und gänzliche Unfähigkeit, etwas Flüssiges zu schlucken, während feste Speisen jetzt in geringer Menge ganz langsam geschluckt werden können.

Linke Gesichtshälfte faltenlos und vollständig gelähmt, starker Lagophthalmus.

Es besteht rollständige Blicklähmung nach links:

Linkes A uge rechts von der Mittellinie stehend, kann nicht im geringsten nach links bowegt werden, wohl aber noch weiter nach rechts, ebenso nach oben und unten.

Rechtes Auge steht in der Mittellinie, kann nicht nach links bewegt werden, wohl aber nach rechts, oben und unten.

Beim Convergenzversuoh tritt das rechte Auge etwas über die Mittellinie nach links, was durch den Versuch, nach links zu blicken, nicht erreicht worden kann.

Bei den Bewegungen der Augen nach rechts, oben und unten besteht otwas Nystagmus.

Pupillen gleich, reagiren sowohl auf Licht wio auf Convergenzanstrengung. Ophthalmoskopisch keine wesentliche Veränderung, vielleicht etwas Abblassung der temporalen Papillenhälfte.

Active Entfernung des Unterkiefers vom Oberkiefer geschieht mit geringer Kraft und der Unterkiefer wird dabei $\mathrm{nach}$ links verschoben. Beim Zubeissen der Kiefer ist deutlich zu füblen, dass der linke Masseter und Temporalis weniger angespannt wird als der rechte.

Zunge weicht etwas nach links ab, doch soheint dies grösstentheils von der Versohiebung des Unterkiefers herzurühren.

Gau mensegel hängend, und zwar links mehr als rechts; beim Phonjren wird die rechto Seite etwas, die linke gar nicht gehoben. Gaumen- und Rachenreflex beiderseits fast ganz fehlend.

Sensibilität des Gesichts. Es worden zwar beiderseits alle Reize wahrgenommen, jedoch in der ganzen linken Gesichtshälfte sowohl Berührung wie Stich weniger stark empfunden, als rechts. Auch für "Kalt" besteht links eine deutliche Abstumpfung, während "Warm “ links stärker empfunden wird als rechts. Auch auf der Schleimhant der linken Mund-, Zungen- und Nasenhälfte ist die Empfindung abgestumpft.

Geschmack auf den vorderen zweiDritteln der linken Zungenhälfte für Sauer, Süss, Bitter, Salzig fast fehlend, rechts normal.

$\mathrm{Hörschärfe}$ auf dem linken Ohr ebenso wie auf dem rechten nicht herabgesetzt,

Sprache nicht erheblich gestört, hat aber immer etwas nasalen Klang und ist etwas undeutlich articnlirt. Stimme nicht heiser. Laryngoskopisohe Untersuchung gelingt wegen der Sohwierigkeit der Mundöffnong und des Tief- 
standes des Kehldeckels nur mit Schwierigkeit; es ergiebt sich, dass das linke Stimmband weniger beim Phoniren angespannt wird als das rechte.

Extremitäten. Die Bewegungen der Arme und Beine werden beiderseits mit gleicher Kraft ausgeführt; es bestebt keine Ataxie und keine Sensibilitätsstörung. Patellarreflex beiderseits gleich stark.

Elektrische Untersuchung. Faradisch rom Nerv. facialis aus Contraction rechts bei $140 \mathrm{Mm}$. R. A., links bei $130 \mathrm{Mm}$. Orbicularis palpebr. direct rechts und links 155, Frontalis reohts 140, links 135, Wangenmuskeln rechts 150, links 140, Orbioularis oris und Kinnmuskeln beiderseits 145 . Galvanisch kein quantitativer Unterschied nachweisbar, wohl aber leichte Zuckungsträgheit links.

Masseter faradisch rechts 130 , links $125 \mathrm{Mm}$. R. A. Temporalis beiderseits 120 R. A. Durch Bestreichen der seitlichen Halspartien mit der einen oder anderen Elektrode des constanten Stromes lassen sich dentlich Schluckbewegungen auslösen.

Die elektrische Untersuchung der Extremitäten ergiebt normalo Verbältnisse.

Verlauf. Nachdem zunäohst vergeblich versucht war, die Patientin durch langsames Schlucken kleinster Portionen za ernähren, wurde zur Ervährung mittelst Schlundsonde gegriffen, was ohne Schwierigkeit gelang. Von da an hörte das quälende Durstgefühl auf. Es wurden ausserdem täg. lich galvanische Scbluckbewegungen ausgelöst, worauf nach 5 Tagen die Fähigkeit, selbstständig Flüssigkeiten zu schlucken, sich wiederherstellte und die Ernährung eine Zeit lang gut von statten ging. Das Kauen war jedoch dauernd mit Schwierigkeiten verknüpft und gesohab fast ausschliesslich mit der rechten Seite.

20. Februar 1891. Seit zwei Tagen ist die Blickläbmung insofern noeh stärker geworden, als beide Bulbinunbeständig nach rechts in den Augenwinkeln eingestellt sind bezw. durch nystagmusartige Zuckungen dahin gezogen werden. Der recbte Balbus wird nur unter grosser Anstrengung für kurze Zeit nach der Mittellinie gebracht, der linke kann gar nicht aus dem Augenwinkel herausgebracht werden. Beim Versueh, nach links zu blicken, treten jetzt gekreuzte Doppelbilder auf. Pupillenreaction normal. Augenhintergrund frei.

19. März 1891. Patientin klagt seit einigen Tagen über Schwächegefühl im rechten Arm, und dass ihr der kleine Finger der rechten Hand jetzt häufig einschlafe. Objectiv ist zu constatiren, dass die rechte Sehulter etwas hängt, und dass sowohl die Erbebung des Arms wie die Bewegung des Vorderarms and der Hand mit geringerer Kraft ausgefübrt werden als links. In der Bewegung des Beins besteht keine Differenz.

Die Sensibilitätsprüfung ergiebt, dass am ganzen rechten Arm u ud der rechten Rumpfhälfte leise Pinselberührungen oft nioht e mpfunden und mangelhaft localisirt werden. Nadelstiche werden weniger dentlich empfunden als links. Im rechten Bein besteht keine Sensibilitätsstörung. A taxio ist nicht nachwoisbar.

Von Mitte April 1891 an musste sie wieder regelmässig mit der Sonde 
ernährt werden, da bei einem Schluckversuch ein heftiger Erstickungsanfall mit fadenförmigem Puls eingetreten war.

Wiederholt waren anch Schwindelanfälle notirt. Ferner hatte der näselnde Charakter der Sprache zugenommen und die Stimme war schwächer geworden.

23. Mai 1891. In lotzter Zeit wiederholtes Erbrechen. Klagen über Schmerzen in den Armen und Beinen.

Befund am rechten Arm wie früher. Dynamometer rechts 50 , links 75. Ferner ergiebt sich jetzt, dass im linken Bein die grobe Kraft etwas abgenommen hat, und dass hier eine analoge Sensibilitätsstörung besteht wie im rechten Arm. Letztere reicht ungefäbr bis zur Nabelhöhe herauf and tritt hier auf die rechte Seite des Rumpfes über. Augenbefund wi $i$ früher. Sebschärfe rechts $=1$, links $=1 / 2$.

Im Norember 1891 wurde bei erneuter Aufnahme des Status in den meisten Beziehnngen der gleiche Befund wie früher erhoben. Die Anästhesie der linken Gesichtshälfte hatte sich etwas vermindert, die Geschmacksempfindung auf der linken Zungenhälfte war um etwas besser geworden. Gesicht nicht mehr so stark nach rechts verzogen, Facialislähmung jedoch im Wesentlichen ungeändert. Faradisch links die Contractionen erst bei $1 \mathrm{Ctm}$. geringerəm R. A. eintretend als rechts und dann noch schwach, während rechts bereits tetanische Verzerrung des Gesichts eintrat. Galvanisch kein quantitativer Unterschied, aber links ausgesprochene Zuckungsträghoit in allen Muskeln. Masseter rechts bei mittlerer Stromstärke faradisch gut erregbar, links nicht in fühlbarer Weise contrahirt. Temporalis bei gleicher Reizung rechts stark, links nur geringer contrahirt.

Schlucken von festen Speisen mühsam, aber nicht mehr za Erstickungsanfällen führend. Schlucken von Flüssigkeiten meist gut. Zuweilen Regurgitation durch die Nase.

Während des Winters 1891/92 bliebt der Zustand im ganzen ungeändert, nur schwand die frühor constatirte Schwäche und Sensibilitätsstörung des linken Beins vollständig, während der rechte Arm dauernd die früheren Verhältnissebot, d. h. eine leichte Abnahmedergroben Kraftund geringe Sensibilitätsverminderung, niemals aber eine Andeutung ron Ataxie. Der Ernährungszustand und das Gesammtbefinden besserte sich in dieser Zeit.

Im Apil 1892 wurde Verbreitung der Sensibilitätsstörung a uch auf die rechte Unterextremität constatirt, sie blieb jedoch bis za Ende überall nur eine unvollständige, indem eine Abstumpfung der Berührungs- und Schmerzempfindung bestand, kein Ausfall derselben.

Im Mai 1892 wurde notirt, dass die $Z_{w}$ angsstellung des linken Anges nach dem Nasenwinkel etwas nachgolassen hatte, ebenso die Stellung des rechten Auges nach dem äusseren Augenwinkel, und dass nan auch die active Beweglichkeit beider Augen nach rechts otwas vermindert war. Das rechte konnte jetzt wieder bei Convergenzanstrengung eine Spor über die Mittellinie nach links bewegt werden. 
17. Mai 1892 nahmen die Schlingbeschwerden wieder zu, die Patientin verschluckte sich öfter, hatte wiederholt Schwindelanfälle und Erbrechen.

Am 3. August trat Fieber ein mit starken Athembeschwerden. Es zeigte sich Dämpfung und Bronchialathmen über dem unteren Theil der rechten Lunge. Sebr erschwerte Expectoration, völlige Unfäligkeit, zu schlucken.

Am 6. August 1892 erfolgte der Tod.

Eine Recapitulation der Symptome ergiebt Folgendes: In etwas mehr als zwei Jahren tödtlich verlaufene Erkrankung. Erstes Symptom linksseitige Facialislähmung; einige Monate später Schwindel, Erbrecben und Schluckstörung, was nach acht Tagen wieder nachliess, um ein Vierteljabr später abermals aufzutreten. Zu dieser Zeit zuerst subjectiv Sehstörung bemerkt, die wahrscheinlich von der bald daranf constatirten Lähmung der Blickbewegungen nach links abhing. Gleichzeitig mit dieser conjugirten Augenmuskellähmung wurde Schwächeder linken Kaumuskeln und Anästhesie im Bereich des linken Trigeminus festgestellt. Etwas später Schwäche und Sensibilitätsverminderung im rechten Arm, was bis zuletzt anhielt; weiterhin vorübergehend die gleichen Erscheinungen im linken Bein, dann dauernd Sensibilitätsabstum pfung im rechten Bein. Die Lähmung der Blickbewegung nach links bis zum Tode anhaltend; in der letzten Zeit auch eine Sch wa che der Blickbewegung nach rechts hinzugetreten. Psychische Functionen bis zuletzt normal. Mehrmals Obnmachten, häufig Erbrechen, niemals Krampfanfälle. Staungspapille feblend.

Die Diagnose war auf eine Neubildung in der Ponsgegend gestellt worden, und zwar wurde angenommen, dass die linksseitige Facialislähmung in Verbindung mit der conjugirten Deviation der Augen nach rechts anf einen Tumor hinweise, der von der Gegend des linken Facialis- und Abducenskernes seinen Ausgang genommen habe. Capitalwärts musste sich der Herd bis in die Trigeminusgegend erstrecken, spinalwärts war die Betheiligung des Glossopharyngeus wegen der nachgewiesenen Geschmacksstörung anzunehmen. Ueber die Natur der Neubildung war ein sicheres Urtheil nicht zu gewinnen; tuberculöse Belastung bestand nicht. Trotzdem auch kein Anhalt für Syphilis vorlag, wurde doch immer wieder die Möglichkeit einer occulten Infection erwogen und daher eine Zeit lang Jod- und Quecksilberbebandlung angewendet. Es schien danach eine Besserung einzelner Symptome einzutreten, so dass der Verdacht verstärkt wurde. Die Section hat aber ergeben, dass er völlig nnbegründet war, indem die Geschwulst selbst sich als reines Gliom erwies und die Untersuchung der übrigen Organe keinerlei Zeichen von Syphilis ergab. 
Sectionsbefund. Bronchopneumonische Infiltration im rechten Unterlappen. Langenödem. Keine erheblichen älteren Veränderungen der Lungen. Herz und Unterleibsorgane normal. Gehirnhänte sowohl an der Convexität wie an der Basis von normaler Beschaffenheit, ebenso das Gehirn selbst, dessen Windungen nur mässig abgeplattet erschienen. Das kleine Gehirn war nach oben gedrängt und unter demselben erschien eine grosse, aus dem Boden des vierten Ventrikels heraustretende Geschwulst von granweisser, stellenweise etwas röthlicher Farbe, welche cerebralwärts bis in den Aquaeductus Sylviizu verfolgen war und sich spinalwärts bis in den Calamus scriptorius erstreckte. Die Geschwulst lag im Wesentlichen in der linken Seitedes Ventrikelbodens, sehien das Ependym des Ventrikels nirgends zu durchbrechen, sondern nur emporzuwölben, hatte die noch überall erkennbare Raphe stark nach rechts verdrängt and zeigte in der Gegend ihrer stärksten Ausdehnung, welche etwa dem spinalen Brückenabschnitte entsprach, mehrere tiefe Einkerbungen. Das spinale Ende der Geschwulst trat wie ein schmaler Zapfen aus der medialen Seite des linken Corpus restiforme hervor.

Die Abbildung Fig. 1 (Taf. XIII.), welehe nach dem bereits einigermassen erhärteten Präparat gezeichnet wurde, giebt eine naturgetreve Darstellung der makroskopischen Verhältnisse. Zu bemerken ist noch, dass auch auf der basalen Seite die linke Brückenbälfte leicht vorgewölbt und seitlich verzogen war, und dass der Trigeminus sowie der Facialis und Abducens dieser Seite etwas abgeplattet und durchscheinend erschienen, während an den übrigen Gehirnnerven beider Seiten, insbesondere anch an Oculomotorius and Trochlearis makroskopisch keine Veränderungen zu bemerken waren. Leider wurde die mikroskopische Untersuchung dieser Nerven in frischem Zustande, da die Obduction in die Zeit meiner Abwesenheit während der Ferien fiel, verabsäumt. Die Gefässe an der Basis des Gehirns zeigten keine Anomalien.

Durch einen cerebralwärts von der hinteren Commissur gelegten Fron- talschnitt, welcher makroskopisch normale Verhältnisse ergab, wurde der Gehirnstamm getrennt und das ganze Präparat ohne Eröffnung des Aquaeductus Sylvii mit Erhaltung des durch einen sagittalen Schnitt auseinandergelegten Kleinhirns in Müller'scher Flüssigkeit gebärtet. Die Erhärtang gelang so gut, dass eine vollständige Schnittserie, vom unteren Ende der Medulla oblongata angefangen, bis in die Gegend des Pulvinar angefertigt werden konnte.

Das Studium diesor Schnitte, wolohe abwechselnd bald nach Weigert oder Pal behandelt, im letzteren Ealle zum Theil mit Carmin nachgefärbt, bald direct mit Carmin oder mit Nigrosin oder mit Hämatoxylin gefärbt wurden, gab über die Verbreitung des Tumors und über seine Einwirknng anf die verschiedenen Abschnitte der betroffenen Gegend Aufsobluss. Leider ging der Uebergangstheil der Oblongata in das Rückenmark und das letztere selbst verloren, so dass über etwaige absteigende Degeneration kein Aufsohluss erhalten werden konnte. 

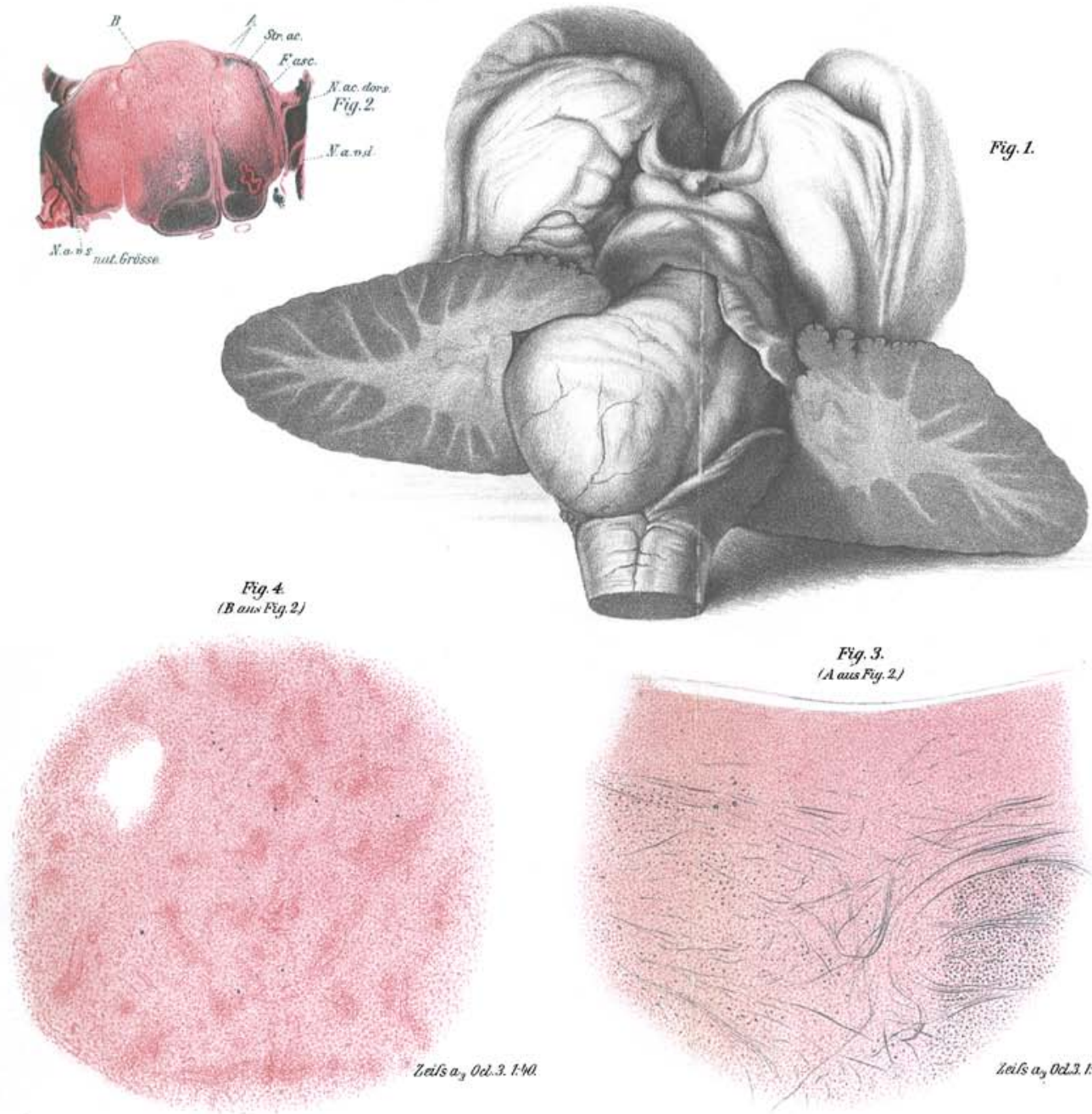

Fig. 3.

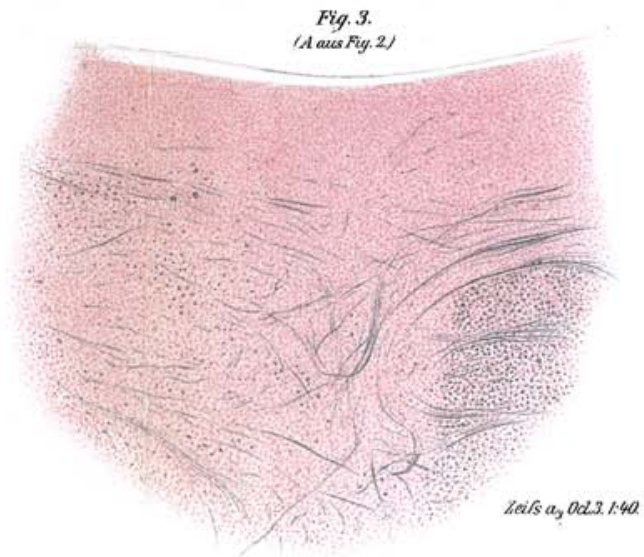


Ein üborsichtliches Bild ron der Ausdehnung des Tumors ist zunäobst aus den folgenden Haptquersehnitten za gewin. nen, welche nach photographischen Aufnahmen ron Pal-Präparaten in natürlicher Grössegezeichnet und zinkographirt warden. Die von dem Tumor eingenommenen Stellen zeichnen sich bei dieser Behandlung durch ihre helle Farbe aus, ähnlich wie die markarme grane Substanz. Aus der blasseren Farbe der begrenzenden Partien der weissen Substanz ist zugleich die Infiltration der nächsten Umgebung und die Auseinanderzerrung der dunkel gefärbten Markfasern zu erkennen, auf die wir zurückkommen. Bei Besprechung der Figuren und der Angabe der näheren Details, unter Heranziehung der dazwischen gelegenen Schnitte, beginnen wir am zweckmässigsten mit dem spinalen Ende des Tumors.

Die ersten A nfänge des Ta mors begegnen uns in einem Schnitte, der nabedem distalen Endedes Hypoglossuskerns geführt ist. Centralcanal noch uneröffnet, aber in der Medianlinie bereits nach rückwärts verlän. gert. Unmittelbarhinter dem linken Hypoglossuskern und ungefähr von gleicher Grösse wie dieser selbst findet sich hier eine helle Stelle, welchewie eine Verdickung des den Centralcanal umgebenden Ependyms erscheint, den Canal aber in einen nach rechts convexen Bogen verdrängt.

Der Hypoglossuskern ist hier anf der linken Seite ebensowie a uf der rechten gut ausgebildet. Seine Zellen und das feine Fasernotz weichen auf den verschieden gefärbten Präparaten nicht von den normalen ab; die austretenden Wurzelbündel sind gleiohfalls normal.

In etwas höher oben gelegenen Schnitten, welche der Figur 1 entsprechen, sind die Verhältnisse im Ganzen dieselben. Es entsteht aber hier schon der Eindruck, als ob alle Theile der linken Oblongatahälftewiedurch $\theta i n \theta n$ von innen her wirkenden Druck otwas a u seinandergezerrt, die der rechten Hälfte etwas comprimirt wären. So ist der linke Hypoglossuskern deutlich grösser und sein Fasernetz lichter als auf der rechten Seite. Die Zellen selbst treten auf der. linken Seite deutlicher hervor, sie sind weiter voneinander entfernt, grösser und zeigen in Carminpräparaten aufallend helle Kerne und unregelmässige and kurze Fortsätze, während die Zellen des rechten Hypoglossuskerns mehr zusammengedrängt erscheinen, dunkler gefärbt and deutlich kleinor wie die der linken Seite sind, dabei aber schärfer contourirt und mit langen gut zu verfolgenden Fortsätzen versehen. Die Hypoglossuswurzeln sind links noch vollständig normal, die zwischen ihnen und dem Hinterhorn gelegenen Fibrae arcuatae internae(Schleifenfasern) sind gut ausgebildet, aber in grösserem Bogen ausgezogen, als rechts und daher im Ganzen beller gefärbt.

Hinter dem Hypoglossuskern erscheint bereits beiderseits der sensible Vaguskern, der auf der linken Seite in der Geschwulstmasse aufgeht, indem er vollständig mit kleinen meist ovalen Kernen infiltrirt ist, zwischen denen die Nervenzellen in grossen $\mathrm{Zw}_{\mathrm{w}}$ - 
Ueber einen Fall von Gliom im dorsalen Abschnitt des Pons ete. 627
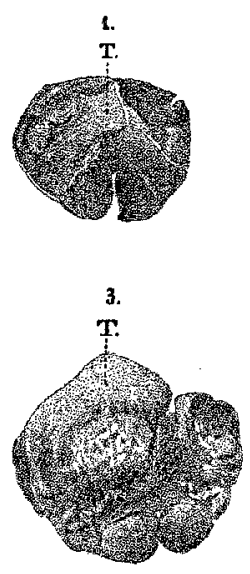

5.
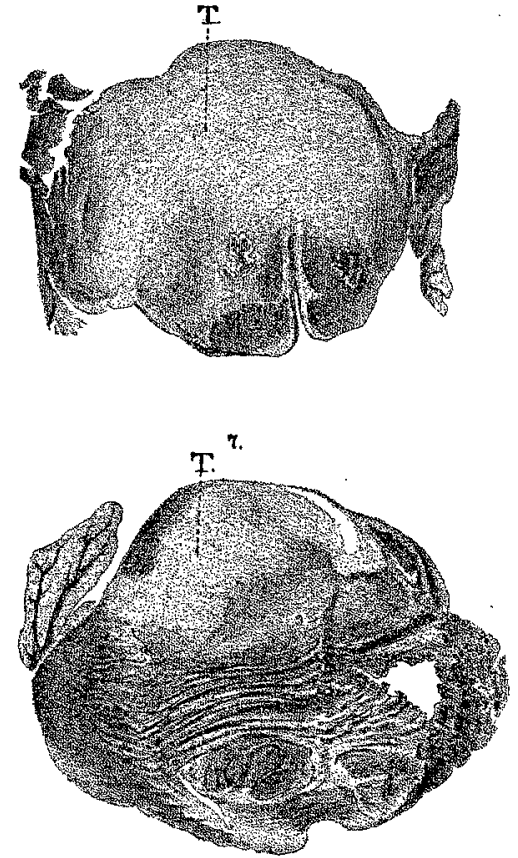
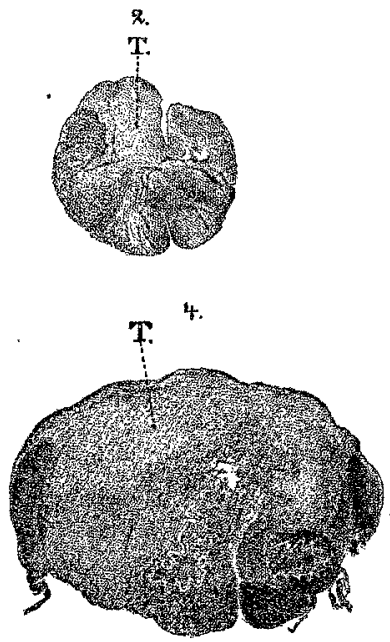

6.

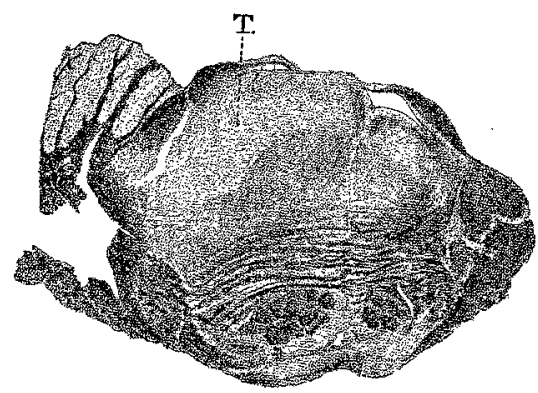

8.

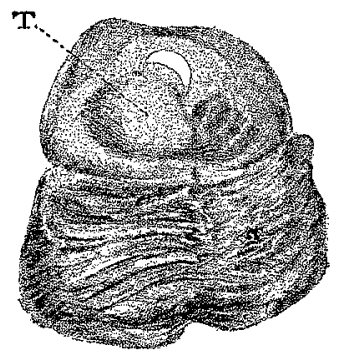

In allen Figuren entspricht der obere Rand dem dorsalen, der untere dem ventralen Rande des Präparats. Sie stellen sämmtlich die spinalen (caudalen) Flächen der betreffenden Schnitte dar, so dass also auch überall die linke Seite der Abbildungen der linken Seite der Präparate entspricht, die rechte der rechten. Mit $\mathrm{T}$ ist in allen Figuren der Tumor bezeichnet. 
sobenräumen, aber noch dentlicherkennbar und im Vergleich zu denen der rechten Seite etwas gequollen vertheilt sind. Dazwischen in Palpräparaten spärliche Reste von feinen Nervenfasern und klumpig verändertem Nervenmark.

Figur 2 stellt einen Schnitt aus der Gegend dar, in welcher eben die Eröffnung des Centralcanals beginnt. Hier zeigt sich, dass der Tumorbereits wie ein etwa erbsengrosser Lappen ans der linken Seitedes Ventrikelbodens herausragt. Er beginnt zunächst wieder in der Gegend des Vaguskerns, erstreckt sich aber lateralwärts in die Hinterstrangkerne, welche direct $n a c h$ a ussen gedrängt sind, deren Structur jedoch trotzder starken Kerninfiltration noch deutlich erhalten ist. Der Vaguskern selbst wird überwiegend aus Kernmassen gebildet, die im Einzelnen den Gliakernen an Grösse und Form entsprechen und zwischen welchen noch reichlich amorphe and feinkörnige Gliasubstanz gelegen ist. Mitten in diesen Massen finden sich vereinzelt grosse, gequollen aussehende Zellen mit deutlichem Kern und Kernkörperchen und einzelnen klampigen Fortsätzen, die offenbar als Ganglienzellen des Vaguskerns anzu-. sehen sind. Nervenmark noch spärlicher wie vorher, aber in einzelnen Resten fast in jedem Gesichtsfeld auffindbar.

Solitäres B ündel an der lateralen vorderen Ecke der Geschwulst als halbmondförmig verzogener Querschnitt mit gut erhaltenen Nervenfasern erkennbar.

Hypoglossuskern ungefähr doppelt so gross wie auf der rechton Soite, stark mit Geschwulstkernen infiltrirt; Zellen zablreich, aber meistgequollen no mit unregelmässigeren Fortsätzen als rechts. Nervenfasernetz heller als rechts. Nervenmark mehr klumpig undabgebröckelt. Austretende Wurzelfasern von normaler Beschaffenheit.

Nach der ventralen Seite zu tritt die Kerninfiltration stärker in die Fibrae arcuata internae ein, und ziehtdieselbenweitausein and $\theta$ r. Die Fasern sind im Ganzen noch gut erhalten, an einzelnen jedoch auch Quellungen und Abbröckelung des Nervenmarks zu bemerken.

Figur 3 (S. 627) entspricht der Gegend, in welcher die Oliven voll ausgebildet sind. Der Tumor bildet als breite, nachoben spitz zulaufende Masse das dorsale Ende der linken Oblongatahälfte. Diese Hälfte ist naheza doppelt so breit geworden wie die rechte, welche in ihrer ganzen Ausdehnung etwas seitlich comprimirt erscheint. Die Raphe ist in der Mitte etwas geknickt, so dass sie einen flachen nach links convexen Bogen beschreibt.

Der Hypoglossuskern der rechten Seite zeigt von rückwärts her eine tiefe Einkerbung, um welche herum er zusammengepresst ist.

Rechter Vaguskern ebenfalls verschmälert. In beiden beginnt in dieser Höhe ebenfalls eine leichte Kerninfiltration.

Der linke Hypoglossuskern ist stark von Geschwulstkernen erfüllt. 
Die Ganglienzellen sind spärlicher, das Nervenfasernetz ist stärker gelichtet and enthält zahlreiche Fasern mit verändertem Mark. In den austretenden Wurzeln vereinzelt solche Fasern. Nervenzellen im linken sensiblen Vaguskern nur noch vereinzeit in veränderten Formen nachweisbar.

Der Nucleus a mbigu us erseheint in dieser Schnitthöherechts deutlich mit gut ausgebildeten Ganglienzellen. Links liegt in der vordersten Partie der Geschwulstmasse zwischen den auseinandertretenden Fibrae arcuatae eine Anzahl von ziemlich grossen, gut erhaltenen Ganglienzellen zerstrent, wahrscheinlich den Nucleus ambiguus darstellend. In gleicher Höhe finden sich austretende Wurzelfasern des Vagus, welche gut erhalten sind, aber direct aus dem Geschwulstyewebe herauskommen.

Solitäres Bündel auf der rechten Seite plattgedrückt, aber aus gut erbaltenen Fasern bestehond. Lin ks ein weit auseinandergezogener Quersehnitt einer Anzahl von Nervenfasern, deren Mark krümelig und gequollen ist.

Fibrae arcuata 0 links stark von Kernmassen durchsetzt, die an einzelnen Stellen förmliche Nester zwischen ihnen bilden. Nervenmark im Ganzen erhalten, zeigt abor vielfach Veränderungen und ist stellenweise unterbrochen.

Schleifenschicht auf der linken Seite ebenfalls stark verbreitert, blass, stark mit Kernon infiltrirt und unregelmässige Markgerinnungen anfweisend, während die rechte Schleifenschicht unverändert erscheint. Linke Olive auseinandergezogen, ihre Markschichten auf Palpräparaten blasser erscheinend als rechts. Linke Pyramide ebenfalls etwas vergrössert und blasser, zeigt aber im Ganzen gut erhaltene Fasern.

In den nächstfolgenden Schnitten erscheint lateralwärts der A custicuskern. Sowohl rechts wie links ist die Umbiegung der letzten Fasern des solitären Bündels in Glossopharyngeusfasern za verfolgen. Der Glossopharyngeuskern, dessen proximalster Thoil links fast ganz aus Geschwulstmasse besteht, kommt gleichfalls zu Ende.

Figr r 4 (S. 627) stellt einen Schnitt etwas oberbalb dieser Gegend dar, entsprechend 0 bersteiner Sohnitt g, Fig. 113.

Der Geschwulstquerschnitt entspricht bier etwa dem einer grossen Kirsche. Raphe stärker nach rechts abgeknickt. Auch in der rechten Hälftedes dorsalen Theiles der Oblongata findetsich hier ziemlich ausgedehnte Geschwulstinfiltration.

Links ist das Corpus restiforme za einer schmalen langen Sichel ansezogen, während es rechts die normale Gestalt zeigt. Dreieckiger Acusticuskern links der dorsalen Seite der Geschwalst entsprechend, fast ganz in ihr aufgegangen. Sowohl die lateralen Abschnittedes N. acusticus (N. cochleae) als die medialen (N. vestibuli) treten in Weigert- ond Palpräparaten als compacte dunklo Faserzüge aus. Je mehr man sie aber nach ihren Ursprungsstellen in dem Geschwalstgewebe verfolgt, um so mehr treten Veränderungen des Marks hervor, das sich klumpigzusammenballtund dessen un- 
regelmässige kugelige und körnigo Reste schliesslich an Stelle der nicht mebrzu verfolgenden Fasern treten.

Am dorsalen Ende der Raphe tritt beiderseits das hintere Längsbündel auf, das aber links nur aus weit auseindergedrängten, meist krümlig veränderten Fasern besteht, während es rechts besseraus gebildet ist, jedoch anch Kerninfiltration aufweist. Der unmittelbar angrenzende Theil der Schleife ist beiderseits stark infiltrirt und enthält links fast gar keine Nervenfasern. Dagegen ist die noch woi ter ventralwärts gelegene Partio der Schleiferechts vollkommen frei, links wenigstens in ihrem ventralen Drittel erhalten, wenngleioh auch hier mit Kernen infiltrirt. Pyramide links etwas verbreitert und blasser als reobts.

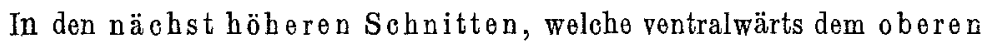
Ende der Olive entsprechen, während sie dorsalwärts bereits in die $\mathrm{Ge}$ gend des Facialis fallen, (es war an dieser Stelle die Theilung des Präparats in zwei zu den Serienschnitten verwandte Blöcke erfolgt; die Sohnittrichtung fiel in der folgenden Serie nicht genau in die gebräuchliche Frontalebene, sondern in eine mehr nach vorwärts geneigte Ebene), zeigt sich, dass der rechte Facialiskern gut entwiokelt ist, der linke dagegen ganz in der Geschwulstmasse a ufgeht, so dass es nur zweifelhaft gelingt, einzelne aufgeblähte, fortsatzlose Zellen, die ihm vielleicht angehören könnten, aufzufinden.

Das Facialisknie ist rechts gut ansgebildet, während es links als verzogener, nur wenige und fast nur veränderte Fasern enthaltender Querschnitt erscheint.

Fig ur 5 (S.627), nebenstehend in nicht ganz 2 facher Vergrösserung wiedergegeben, entspricht den obersten Sohnittreihen dieser Gegend. Rechts zeigt sich bereits die als compactes Bündel austretende Facialiswurzel, die mikroskopisch nicht verändert erseheint. Links lassen sich an der Peripherieder hier in zwei Lappen getheilten Geschwulst ein$z e \ln \theta$ tangential verlaufende, wahrscheinlich der austretenden Wurzel angehörige Fasern verfolgen, die aber stark verändertes Nervenmark zeigen und an verschiedenen Stellen unterbrochen sind. Die (in anderen Sohnitten) an der ventralen Seite aus der Geschwulstmasse austretenden Facialisw urzeln zeigen ebenfalls starke Veränderungen und erhebliohen Schwund des Nervenmarks. Eine directe Verbindung zwischen ihnen und den erst erwähnten tangentialen Fasern ist nirgends nachzuweisen.

Vom Abducenskern ist links in keinem Schnitt auch nur ein Rest aufafinden; er ist offenbarganz in der Gesebwulst a ufgegangen. Wurzelfaserndes Abducens sind inderdorsalen Brückenhälfte ebenfalls in keinem Sehnitt za finden; dagegen erseheinen in der ventralen Brückenhälftevielfach mebr oder weniger degenerirte, aucheinzelne ganz gut erbaltene Faserstücke. In 
den nach dem Austritt quer getroffenen Wurzeln zeigt sich starke Degeneration, die nur wenige markhaltige Fasern übrig gelassen hat.

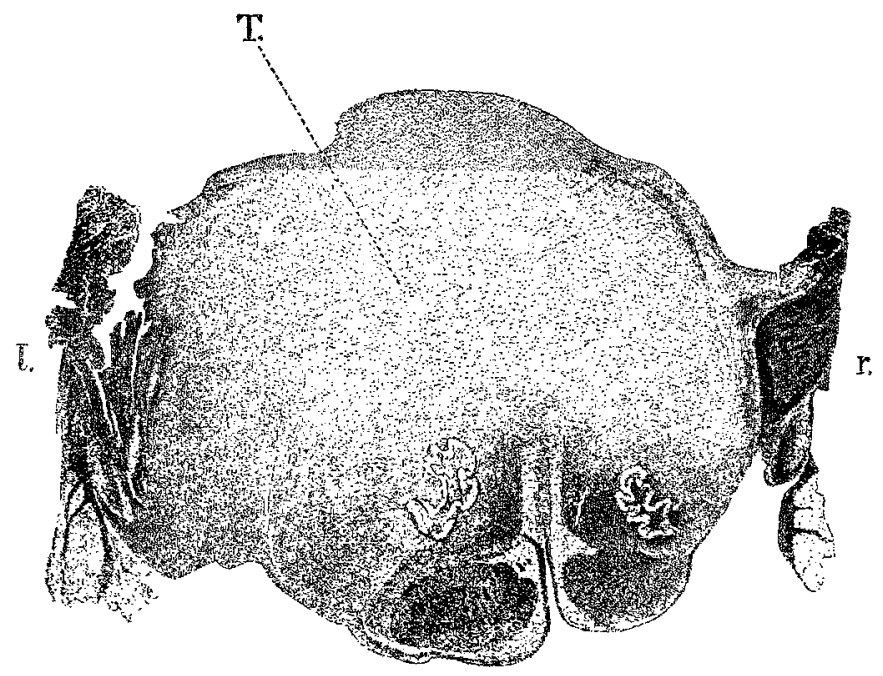

Figar 5.

Der rechte Abducenskern ist durch eine Reihe von Schnitten za verfolgen. Er enthält deutliche, aber bereits klumpig veränderte und fortsatzarme Ganglienzellon, die in zahlreiche Geschwulstkerne wie oingobettet sind. Die Wurzelfasern sind hier sowohl im dorsalon wie im ventralon Brückenabschoitt in compacten Zügen zu verfolgen, zum Theil ebenfalls mit Kernen infiltrirt, Die Nervenfasern sind im Ganzen gut erhalten, zeigen aber stellenweise doch schon auffallende Markgerinnungen.

Dor (grosszelligo) Dorsalkern dos N. acusticus ist in den Präparaten dieser Schnitthöhe rechts überall gut ausgebildet and frei von Infiltration, links ist er ebenfalls erhalten, aher stark nach aussen gedrängt. Es findet sich hier zwischon den zahlreichen gut erhaltenen Ganglienzellen ziemlich starke Kerninfiltration. Die austretende Wurzel des Acusticus zeigt keine stärkero Veränderúng, sie erscheint abor in ihren beiden Abschnitten (N. cochleae and vestibularis) etwas blasser als reobts. Der ventrale Acusticuskern ist sowohl links wio rechts lateralvom Nervus cochleaein normaler Ausbildung naeh. woisbar. Links isterabor starkseitlich comprimirtund in dorsoventraler Riohtung in die Länge gezogen (siehe auch die Abbildung Taf. XIII, Fig. 2).

Die linke aufsteigende Quintuswarzel, wolche schon in den Schnitten aus der Oblongata überall stark seitwärts verschoben nnd verzogen erschien, ist auch in den jetzt in Frage kommeoden Sohnitten links an der 
medialen Seite der Acusticuswurzel als blasser (in Pal- und WeigertPräparaten), länglich ausoinandergezogenor Querschnitt zu sohen, der der Hauptmasse der Geschwulst aussen anliegt und stellenweise von Keruen durohsetzt wird. Rechts erscheint dieselbe normal.

In der nächstfolgenden Schnittreibe erscheint der sensible und motorische Trigeminusern and die austretenden Trigeminuswur. zeln. Die Kerne sind rechts in vollkommen normaler Weise ausgebildet, dio Wurzelfasern frei von Veränderungen. Links dagegen ist jedenfalls der ven tralo (motorische) Korn vollstäpdig in der Geschwulst aufgegangen und in keinem Präparat mehr deatlich nachweisbar. Auch der dorsale (sensible) Kern ist stark infiltrirt und nur noch duroh einzelnerudimentäre, ron Kernen umgebene Ganglienzellen an-

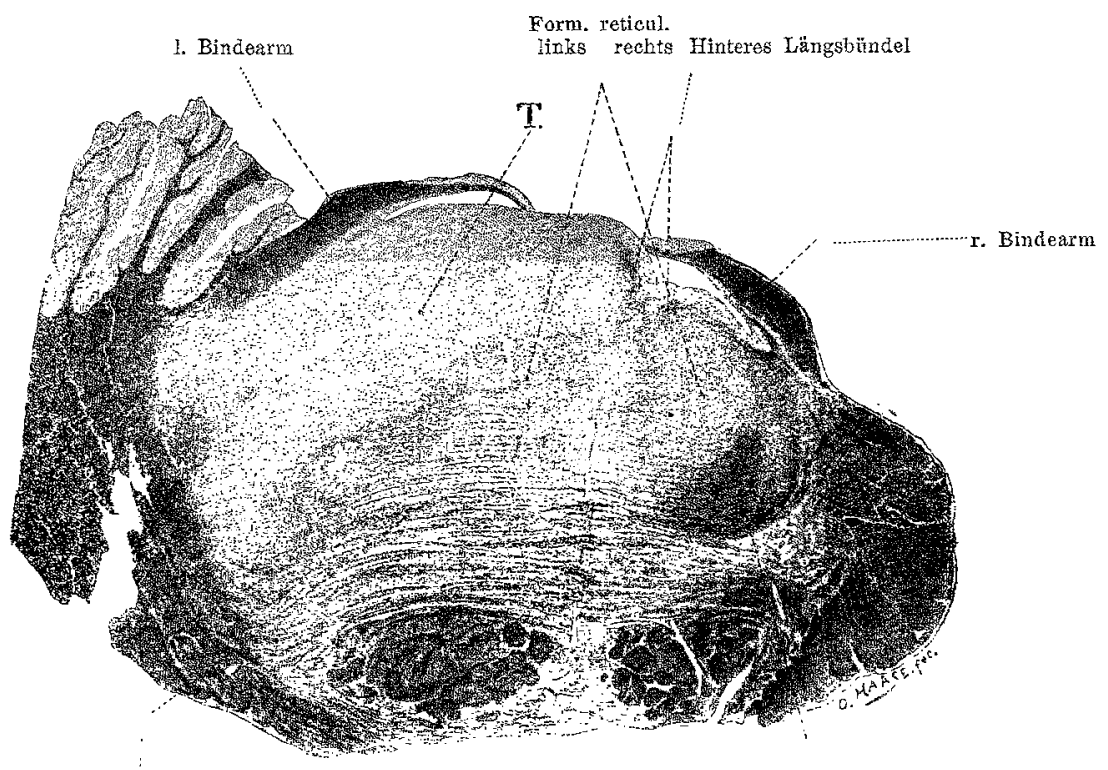

tinke mediale Schleife

Figur 6 .

rechte mediale Schleife

gedeutet. Die austretenden Wurzeln, und zwar besonders stark die motorisohen sind blass und zeigen starken Markzerfall.

In den Präparaten dieser Schnitthöhe (Figur 6, S. 627 und vergrössert S. 632) hat dio Gosohwulstboreits stark an Mächtigkeit verloren; sie liegt ausschliesslich auf der linken Seite, und zwar ganz lateral ir der Eeke des vierten Ventrikels. Ihr Quersohnitt bildet bier einen stumpfen, mit der Convexitätdorsalwärts gewendeten Haken, dessen laterale Spitze in zwoi geschweifte Enden ausläuft. Die linke Hälfte der Haubengegend ist noch immer um mehr als das Doppeltebreiter wie die rechte. Diedurch den Tumor bedingte 
Verschiebung der Theile tritt namentlich dorsal an den Bindearmen zu Tage, welche beide zu schmalen Sicheln ausgezogen sind, zwischen denen das Velum medullare anticum als eine ganz schmale Brücke den Ventrikel und dann den Aquaeductus überdeckt.

An der concaven Seite der Geschwulst liegt links die Formatio retiealaris, deren Gefüge im Ganzen wohl erkennbar ist. Nur ist sie im Verbältniss zu der rechten vollständig normalen Partie im Ganzen vergrössert, a uf Palpräparaten bedeutend blasser als rechts und zeigt sich a u Carminpräparaten diffus von Kernen infiltrirt. An vielen ihrer Nervenfasern finden sich unregelmässige Markgerinnungen.

Die Schleife erscheint in dieser Gegend beiderseits als horizontaler, den dorsalen Querfasern der Brücke aufsitzender Querschnitt, rechts in normaler Ausdehnung und Färbung, links dagegen ist derselbe nicht nur wie hier die ganze Haubengegend nm ungefähr das Doppelte in die Breite gezogen, sondern auch im Ganzen blasser als rechts und in seiner ganzen lateralen Hälfte vollständig durch den anteren Haken der Geschwulst durchbrochen. Mikroskopisch fin. den sich zwar in dieser Gegend noch viele offenbar zur Schleife gehörige Faserquerschnitte im Geschwulstgebiet, viele zeigen aber unregelmässige Gerinnung und Abbröckelung des Marks.

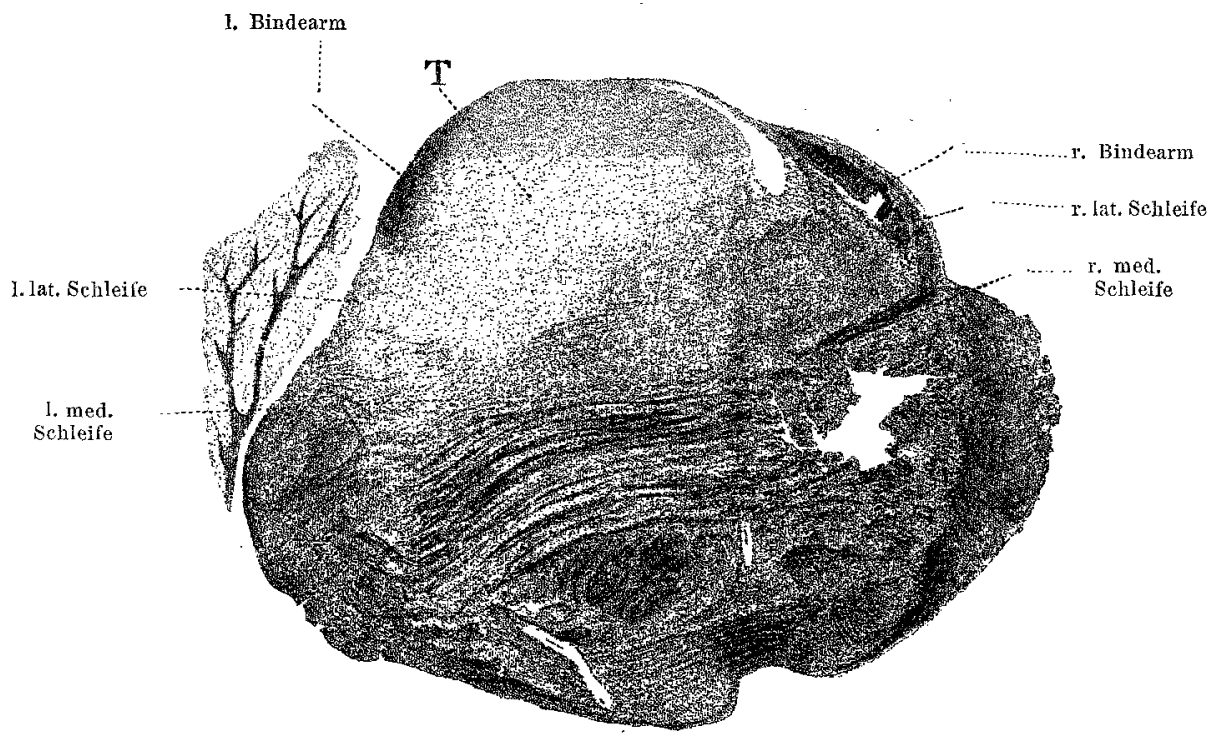

Figur 7.

Figur 7 (S. 627 und vergrössert S. 633) stellt einen Schnitt etwas spinalwärts von der Austrittsstelle des Trochlearis dar. Go- 
schwulst wievorherals stumpfer Haken der Formatio reticularis a usitzend, diese selbst noch etwas verbreitert und infiltrirt. Am lateralen Rande der Geschwolst der linke Bindearm aufsitzend, in die Längegezogen und blasser als der rechte, mikroskopisch unter seinen Fasern ziemlich viele mit zerbröckeltem Mark.

Laterale Schleife rechts bereits als oompacter Zug nach a us son vom Bindearm in die Höhe steigend. Links ist dieselbo vollständig vomTumor infiltrirt, mikroskopisob in Pal- and Weigertpräparaten nur durch spärliche Markreste nachweisbar.

Das Gleiche gilt für den äusseren Theil der linken medialen Schleife, während der in nere $(\operatorname{medial} \theta)$ Theil derselben besser erhalten, aber ebenfalls blass und mit zahlreichen krämeligen Markgerinnungen versehen ist. Die recbte mediale Schleife ist normal.

Das hintere Längsbündel, das in den tieferen Schnitten zwar überall nachweisbar war, aber links in sehr reducirter Form und mit stark degenerirten Fasern, erscheint auch in Figur 7 noch durch den Tumor nach der Dorsalseite hin verzogen und blasser als das rechte; Faserdegeneration nooh deutlich, abor weniger stark.

Figur 8 (S. 627 und vergrössert S. 634) entspricht einem Schnitt durch die hinteren Vierhügel, der schon nahe hinter dem vorderen Geschwulstende gelegen ist. Die Geschwulst ist hier nur noch linsen-

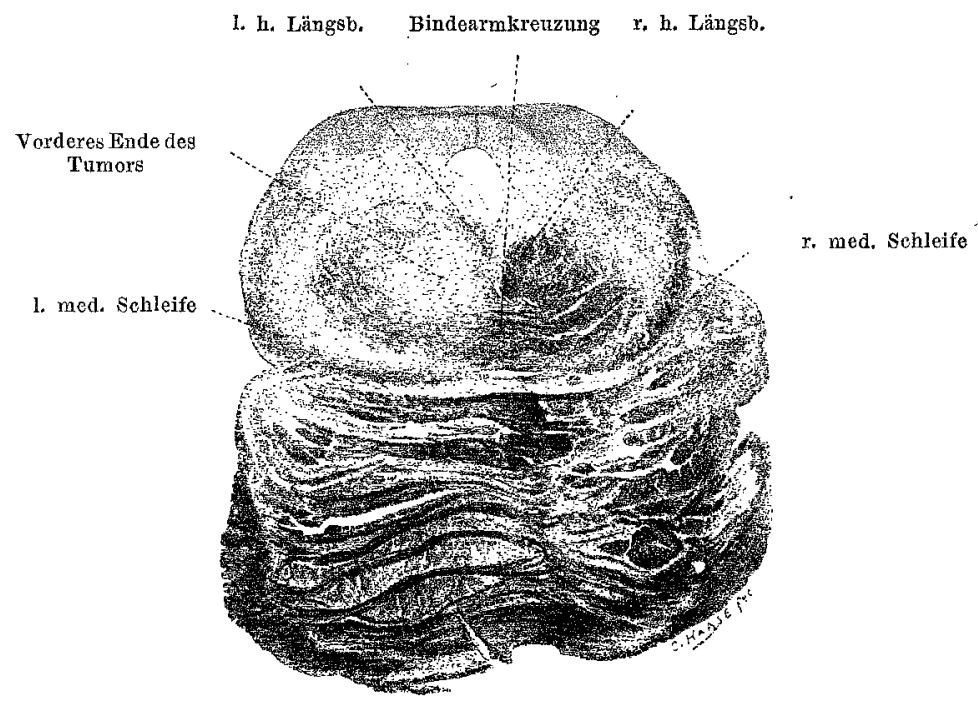

Figur 8.

gross und ron runder Form. Sie schiebt sich in der hier beginnenden Bindearmkreuzung zwischen den linken Birdearm und die Gegend der Raphe ein, wird aber ventralwärts schon von sich kreazenden Bindearmfasern umgeben. Zur Ergänzong sei bemerkt, dass in den 
nächstfolgenden Sohnitten die rascheweitere Abnahmeder Gesohwulstza verfolgen ist, so dass sie bald nurnochwie oin Stecknadelknopf dievon links her eintretenden Bindearmfasern nach oben and anten auseinanderziebt, um dann in der Gegend der vorderen Vierhügel vollkommen zu verschwinden.

Die linke laterale Schleife wird auf Figur 8 in ibrem letaten in der linken Vierbügel übergehenden Theil als gut erhaltenes Faserbündel sichtbar. Auch die mediale Schleife ist hier links in allen Theilen gut ausgebildet, nur immer nooh etwas in die Breite gezogen und um ein Geringes blasser als die rechte.

Das linke bintere Längsbündel wird in Figur 8 noch durch die convexe Seite des Tumors nach oben gezerrt; es stellt $\theta$ inen gut gefärbten, mikroskopisch durchaus normal erscheinenden Faserzug dar.

An seiner dorsalen Seite erscheint in dieser Schnitthöhe links wie rechts in einer Aushöblung der gut ausgebildete Troohleariskern, dessen Ganglienzellen links von annähernd gleicher Form und gleichem Aussehen sind wie rechts. Auch das foine Fasernetzim Kern tritt in Weigertpräparaten beiderseits gleich deutlich hervor und das Gleiche gilt von den auf der lateralen Seite den Kern verlassenden Wurzelbündeln des Trochlearis. In den tieferen Schnitten war bereits die Kreuzungstelle des Troohlearis im Velum medullare anticum zu sehen mit den nach beiden Seiten in gleicher Schwärze und Dicke anstretenden Nervi trochleares. Ebenso liessen sich die aufsteigenden Wurzelbündel beider Nerven bis in die Gegend der Fig. 8 beiderseits als normale Querschnitte verfolgen.

Von den weiter cerebralwärts gefährten Schnitten wurde keine $\mathrm{Ab}$ bildung beigefügt, weil hier vollkommen normale Verhältnisse bestehen mit Ausnabme einer ganz geringen Verschiebung des Querschnittes des hinteren Längsbündels, welches links überall wie etwas dorsalwärts gehoben erscheint, ohne dass aber hier noch etwas von Kerninfiltration nachweisbar wäre.

Die 0oulomotoriuskerne haben sich auf derganzen Schnittserie beiderseits als normal erwiesen. Weder bezüglich der Menge oder der Form der Ganglienzellen, noch bezüglich der Ausbildung des Fasernetzes, noch endlich bezüglich der austretenden Wurzeln und der Querschnitte der ausgetretenen Nerven ist zwischen rechts und links ein Unterschied zu erkennen.

Aus der vorstehenden Beschreibung ergiebt sich, dass der Tumor seine grösste Ausdehnung in der Gegend des Facialis- und Abducensursprunges erfahren hatte und dass er hier nicht nur links. auf der Seite seines eigentlichen Sitzes, die Kerne dieser Nerven vollständig zerstört, sondern dass er auch nach rechts die Raphe durchwachsen und den Abducenskern infiltrirt hatte. 
Sowohl in cerebraler, wie in spinaler Richtung beschränkte er sich dann bald ausschliesslich auf die linke Seite und betheiligte hier in ersterer Richtung noch den Trigeminus, dessen motorischer Kern ganz, dessen sensibler grösstentheils zerstört war, während nach dem Rückenmark zu ein Theil des Acusticnskernes, sowie der Glossopharyngeus- und Vaguskern grösstentheils von Geschwulstmasse infiltrirt und zum Theil zerstört waren, der Hypoglossuskern in seinem proximalen Theile ebenfalls starke Veränderungen zeigte, nach dem Rückenmark za dagegen mehr und mehr normal wurde.

Das klinische Bild, das vorher zusammenfassend geschildert wurde, lässt sich aus dieser Verbreitung des Tumors vollkommen befriedigend erklären. Der Beginn mit linksseitiger Facialislähmung entspricht der Thatsache, dass in der Facialisgegend die Geschwulst ibre gröste Ausdehnung erreicht hatte, und es ist daher wohl die Annahme gerechtfertigt, dass in dieser Gegend der Ausgangspunkt der Neubildung zu suchen ist. Ob zuerst der Kern des Facialis oder dessen absteigende Wurzel infiltrirt wurde, ist nicht mit Sicherheit zu bestimmen. Für die erstere Annahme würde der Umstand sprechen, dass die Erscheinnngen der Entartungsreaction im Facialisgebiet verhältnissmässig spät zur Entwickelung kamen, lange Zeit hindurch nur in leichter Mittelform bestanden und bis zuletzt nicht sich zu der schweren Form ausbildeten. Bei primärer Läsion der absteigenden Wurzel hätte im Lanfe von zwei Jahren wohl eine complete Degeneration des Eacialisstammes bis in die Peripherie und damit schwere Form der Entartungsreaction erwartet werden müssen. Darf demnach sicher angenommen werden, dass die anfänglich allein bestehende Facialislähmung eine Kernlähmung war, so würde sie insofern ein besonderes Interesse beanspruchen, als sie dann die Abhängigkeit sämmtlicher Facialisäste, sowohl der oberen wie der unteren vom Facialiskern, entgegen der Ansicht von Mendel, beweisen würde. Der Fall ist aber in dieser Richtung nicht mit Sicherheit verwerthbar, weil bei der eigenthümlichen Natur des Glioms als einer infiltrirten, weit über die Grenzen der direct zerstörten Partie hinausreichenden Geschwulst die Möglichkeit offen bleibt, dass gleichzeitig mit der primären Zerstörung des Facialiskerns wenigstens eine gewisse Kerninfiltration der absteigenden Wurzel und in Folge hiervon eine Leitungsunterbrechung sämmtlicher Fasern eingetreten sein kann.

Von den einzelnen in dem vorliegenden Falle beobachteten Erscheinungen bedürfen noch folgende einer gesonderten Besprechung:

1. Die conjugirte Angenmuskellähmung. Dies Symptom 
ist wohl das charakteristischste für die im dorsalen Brückenabschnitt vorkommenden Läsionen und es ist so häufig beschrieben und in Bezug auf seine Abhängigkeit von den anatomischen Verhältnissen dieser Gegend analysirt worden, dass ich mich auf eine kurze Hervorhebung der Besonderheiten des mitgetheilten Falles beschränken kann. Die gleichzeitige Lähmung dẹs linken Abducens und des rechten Rectus internus war $z$ weifellos erheblich später aufgetreten als die linksseitige Facialislähmung, wahrscheinlich erst einige Wochen vor Beginn der klinischen Beobachtung, als die Kranke eine "Verschleierung des Blicks" bemerkte. Die willkürliche Bewegung beider Augen nach links war jedenfalls beim Eintritt der Kranken in die Klinik bereits vollständig aufgehoben.

Uebereinstimmend mit fast allen analogen Fällen fanden sich die Oculomotoriuskerne und-Wurzeln unversehrt. Der Fall zeigt also zunächst auch wieder, dass die Lähmung des Rectus internus einer Seite durch eine spinalwärts von seinem Kern in der gegenüberliegenden Brückenhälfte sitzende Affection bedingt werden kann, welche gleichzeitig den gekreuzten Abducens lähmt. Die Discussion dreht sich seit der ersten Veröffentlichung von Foville*), welcher sich die wichtigen Untersuchungen von Féréol, Graux, Wernicke und vielen Anderen anschlossen, um die Frage, welches der genauere Verlauf der Bahn für die associirten Seitwärtsbewegungen der Augen sei und in welcher Beziehung der Abducenskern zu derselben stehe. Hunnius**), welcher die in Frage kommenden Verhältnisse am eingehendsten erörtert und durch eine schematische Abbildung illustrirt hat, kommt auf Grund der bis dahin gesammelten Fälle zu dem Schlusse, dass entsprechend dem von Foville aufgestellten Postulat ein solches Centrum für die Seitwärtsbewegung beider Augen im Abducenskern selbst oder in dessen unmittelbarer Nachbarschaft gelegen sei. Die Lähmung der Seitwärtsbewegung könne entweder durch directe Zerstörung dieses Centrums oder durch Unterbrechung der vom Grosshirn herabtretenden, in der Haube verlaufenden Willkürbahn zu dem Centrum bewirkt werden. Für beide Arten der Entstehung liegen in der That anscheinend beweisende Fälle vor. Am häufigsten fand sich der Abducenskern selbstzerstört, so in den Fällen von Féréol, Hallopeau, Wernicke, Graux, P. Meyer, Bernhardt u. v. a.; aus jüngster Zeit

*) Bulletins de la socióté anatomique. 1858.

**) Zur Symptomatologio der Brückenerkrankungøn etc. 1881 . 
ist der Fall von $\mathrm{Kolisch}^{*}$ ) zu erwähnen und ebenso hat sich in meinem Falle eine vollständige Zerstörung des linken Abducenskerns ergeben. $\mathrm{Zu}$ den Fällen, in welchen der Abducenskern nicht betroffen wurde, gehört der von Hunnius mitgetheilte, ferner aus den folgenden Jahren die Fälle von Quiocq, GareI, Senator u. a. Senator*:) nimmt auf Grund seines Falles, in welchem der Herd vom spinalen Ende des Abducenskerns bis nicht ganz zum spinalen Ende des Hypoglossuskerns rejchte, an, dass das Centrum für die Seitwärtsbewegung der Augen peripher (caudal?) vom Abducenskern gelegen sei. Die Beobachtung würde sich jedoch unter Annahme eines im Kern selbst gelegenen Centrums auch so deuten lassen, dass die Willkürbahn zu demselben erst bis in die Gegend seines caudalen Endes spinalwärts verläuft und dann nach dem Kerne umbiegt.

Direct gegen die Annabme, dass im Abducenskern selbst ein Centrum für die Seitwärtsbewegung beider Augen gelegen sein könne, spricht jedoch einer der vonSiemerling****) mitgetheilten Fälle von Ophthalmoplegie. Es ist dies der Fall 8 der betreffenden Serie, in welchem zunächst doppelseitige Abdacenslähmung, später eine vorwiegend einseitige Oculomotoriusparese eintrat. In dem schwächer befallenen Auge blieb gerade der Rectus internus bis zuletzt vollständig frei, während beide Abducenskerne stark atrophisch gefunden wurden.

Wenn hiernach im Abducenskern selbst kein Centrum mit der erwähnten Function liegen kann, so ist die Frage aufzuwerfen, ob überhaupt ein "Centrum" dieser Artim Pons anzunehmen ist und ob nicht die Sache auch so liegen kann, dass die Willkürbahn für den Rectus externus der einen und den Rectus internus der anderen Seite zunächst bis in die Gegend des Abducenskerns spinalwärts verläuft, dann hier umbiegt und theils mit dem Abducenskern in Verbindung tritt, theils cerebralwärts nach dem Oculomotoriuskern hin sich begiebt. Da ein Centrum für die Seitwärtsbewegung der Augen nach den bekannten Versuchen von Ada$m \ddot{c} c k$ schon in den gleichseitigen Vierhügeln anzunehmen ist und da ein erstes Centrum bezw. ein Ort gemeinsamer Innervation der beiden assnciirten Muskeln in der gekreuzten Grosshirnhemisphäre vorhanden sein muss, von welchem aus die Bahn nach den Vierhügeln und dann in die Haube verläuft, so erscheint ein weiteres Centrum in der

*) Wiener klin. Wochenschr. 1893. No. 14.

**) Dieses Archiv Bd. XIV. 1883.

***) Dieses Archir Bd. XXII. Supplementheft. 
Brückengegend wenigstens nicht ohne Weiteres erforderlich*) und sicher durch die bis jetzt vorliegenden Beobachtungen nicht erwiesen.

Ist diese Auffassung richtig, so wird die Blicklähmung in den Fällen von Hunnius, Senator u. s. w. als fasciculäre aufzufassen sein, bedingt durch Leitungsunterbrechung zwischen den motorischen Kernen und der zu ihnen führenden nächsten centralen Bahn; in den zahlreichen Fällen von Abducenskernbetheiligung (Féréol, Wernieke, P. Meyer u. s. w.) würde es sich dagegen um die Combination einer Kernlähmung (Abducens) mit einer fasciculären (Rectus internus der anderen Seite) handeln und die Ursache für die Häufigkeit dieser Combination wäre darin zu suchen, dass die betreffende Bahn bis unmittelbar an den Abducenskern eine gemeinsame ist, und dass erst von hier aus die Bahn zum Oculomotoriuskern isolirt in cerebraler Richtung verläuft. Dass die letztere auf diesem Wege isolirt unterbrochen werden kann, beweist der interessante Fall von Gubler (Gaz. hébdom. 1858 und 1859), in welchem ein Tuberkel, der links unter dem vorderen Abschnitt des Bodens der Rautengrube sass, eine rechtsseitige Internuslähmung bewirkt hatte, ohne die Function des linken Abducens zu betheiligen.

Was die Lage dieser zwischen Abducenskern der einen Seite und Oculomotoriuskern des Rectus internus der anderen Seite rückläufigen (cerebralwärts verlaufenden) Bahn betrifft, so ist sie bekanntlich noch nicht mit Sicherheit ermittelt. Die Wahrscheinlichkeit spricht für dsa hintere Längsbündel, das sowohl mit dem Abducenskern, wie mit dem Oculomotoriuskern in Faseraustausch zu treten scheint. Durch die Thierexperimente von Gud den wissen wir nun, dass ein Theil der Fasern des Oculomotorius aus dem Kern der gegenüberliegenden Seite entspringt. Von verschiedenen Autoren sind diese Fasern auch im Gehirn des Menschen gefunden worden und insbesondere liegt aus neuester Zeit die gewichtige Bestätigung von Kölliker (Handbuch der Gewebelehre, 3. Aufl., 1893) vor, welcher als gekreuzte Fasern diejenigen bezeichnet, welche den am nächsten der Medianebene verlaufenden Nervenbündeln entsprechen. Sollten dies die Fasern für den Internus sein - was aber bis jetzt nicht nachgewiesen ist - so würde eine

*) Zu Gunsten eines solchen würden nur die Beziehungen der associirten Augenbewegungen zu acustischen und tactilen, namentlich rom Trigeminus ausgehenden Reizen sprechen. Es kann daher selbstrerständlich nicht ausgeschlossen werden, dass secundäre Verbindungen der s. v. v. Seitenblickbahn mit den Kernen dieser Nerven bestehen. Aber ebensowohl lässt sich eine Vermittlung durch die Vierhügel vorstellen und so werden also zunächst die Ergebnisse weiterer Forschung abzuwarten sein. 
Kreuzung der oben besprochenen Seitenblickbahn in ihrem Verlauf vom Abducenskern bis zum Oculomotorinskern nicht mehr anzunehmen sein, sie würde vielmehr in den gleichseitigen Oculomotoriuskern verlaufen müssen, um von hier aus den Internus der gegenüberliegenden Seite $\mathrm{zu}$ innerviren.

In unserem Falle war das hintere Längsbündel der linken Seite stark lädirt, das der rechten zeigte in einigen Schnitthöhen Defecte und wies einzelne Fasern mit Markzerfall auf, war aber sonst überall noch deutlich als gesonderter Strang zu erkennen. Hier war für die Blicklähmung nach links schon in der völligen Zerstörung des Abducenskernes und der starken Geschwulstinfiltration seiner ganzen Umgebung die ansreichende Unterlage gegeben; die Unterbrechung des linken hinteren Längsbündels konnte daher für die Symptome nicht weiter in Betracht kommen. Auffallend war aber in diesem, wie in einigen anderen der beschriebenen Fälle, dass während eines Theils des Krankheitsverlanfes das linke Auge stark in den inneren Augenwinkel eingestellt erschien, viel mehr, als dies bei einfacher Abducenslähmung beobachtet wird. Hierfür muss jedenfalls eine Reizung der rechtsseitigen Bahn für den linken Rectus internns verantwortlich gemacht werden. Eine solche Reizung wird leicht verständlich, falls diese Babn in der That im hinteren Längsbündel liegt, da dasselbe in der Nähe der Mittellinie jn unmittelbarer Nachbarschaft der Geschwulst verläuft und durch dieselbe seitlich verschoben und comprimirt wurde.

In den letzten Monaten des Krankheitsverlaufs trat nun insofern eine Aenderung des Bildes ein, als die starke Deviation des linken Auges nach rechts nachliess, so dass dasselbe nur noch wenig über die Mittellinie hinausstand, and als sich gleichzeitig eine Schwäche in der Auswärtsbewegung des rechten Auges bemerklich machte. Es ist wohl mit Sicherheit anzunehmen, dass die in der Umgebung der Zellen des rechten Abducenskerns gefundene Kerninfiltration, die sich auch auf die weitere Nachbarschaft des Kerns erstreckte, die Ursache dieser Parese der Blickbewegung nach rechts gewesen ist, und dass mit ihr zugleich ein Nachlass der Reizung in der linken Internusbahn eintreten musste, zumal da nun auch Veränderungen im rechten hinteren Längsbündel hinzukamen.

2. Sensibilitätsstörungen. Die während des ganzen Krankheitsverlaufs beobachtete Sensibilitätsverminderung im linken Trigeminusgebiet erklärt sich ohne Weiteres aus der Geschwulstinfiltration im Ursprungsgebiet dieses Nerven. Der motorische Kern desselben war vollständig zerstört - daher die Lähmung und Atrophie der linksseitigen Kaumuskeln -, während der sensible Kern 
weitgehende Zellveränderungen zeigte; die spinale Wurzel war zwar erhalten, aber stark abgeplattet und wies stellenweise Markzerfall auf, die absteigende. Wurzel war ebenfalls erhalten, aber ihre Querschnitte erschienen durch die Geschwulstmasse stark nach der Seite hin verschoben, ohne indess Zeichen des Zerfalls darzubieten.

Sensibilitätsstörungen in den Extremitäten wurden bei der Kranken in wechselnder Form gefunden. Zunächst war bei der Aufnabme, also anderthalb Jahre vor dem Tode, die Abwesenheit jeder Sensibilitätsstörung in den Extremitäten constatirt worden. Drei Monate später trat gleichzeitig mit motorischer Schwäche eine Abnahme der Berührungs- und Schmerzempfindlichkeit im rechten Arm und der rechten Thoraxhälfte ein, also gekreuzt mit der Trigeminusanästhesie; nach weiteren zwei Monaten stellte sich die gleiche Erscheinung in der linken Unterextremität und unteren Rumpfhälfte ein, also auf der Seite der Trigeminusanästhesie. Bekanntlich wird die Bahn der medialen Schleife, welche mit den Hinterstrangkernen in gekreuzter Verbindung steht, sowohl auf Grund anatomischer, wie pathologischer Untersuchungen als die hauptsächliche Trägerin der sensiblen Leitung in der Brückengegend angesehen. Fälle mit vollständiger Zerstörung dieser Bahn, wie u. A. namentlich der sehr gut untersuchte Fall von P. Meyer (I. c.), haben denn auch sehr ausgeprägte Sensibilitätsstörungen in der gekreuzten Körperseite ergeben. In Fällen mit nur theilweiser Zerstörung der Schleife sind dagegen, wie aus der neuerdings veröffentlichten dankenswerthen Zusammenstellung von Moeli und Marinesko (d. Archiv, Bd. XXIV) hervorgeht, die Sensibilitätsstörungen zwar regelmässig gefunden worden, aber meistens doch nur in Form von Hypästhesien, nicht von Ausfall irgend einer Empfindungs- oder Gefühlsqualität. So verhielt es sich auch in dem von diesen Autoren selbst mitgetheilten Falle, in welchem aber ausser der Hypästhesie auch noch Reizerscheinungen in Form von Parästhesien bestanden. Anch der seitdem veröffentlichte Fall von Kolisch (l. c.), in welchem die Schleife in ihren zunächst auf die Kreuzung folgenden Theilen erhalten war, mehr cerebralwärts aber starke Degeneration aufwies*), hatte nur Нyp-

*) In der Beschreibung der Schnitte ans dem unteren Brückendrittel wird bemerkt (rechts) "die Schleife fehlt vollständig“. Da aber von der nächstfolgenden Schnittserie aus dem mittleren Brückendrittel bemerkt wird: "Auch in dem Verhalten der Sohleife und des hinteren Längsbündels zeigen diese Schnitte nicht viel Neues. Die Schleife ist wie an den tieferen Schnitten beiderseits stark geschädigt, besonders wieder auf der rechten Seite", so darf 
ästhesie in der gekreuzten Oberextremität ergeben neben schmerzhaften Parästhesien, die sich zu den heftigsten Schmerzen steigerten - worin der Autor wohl mit Recht ein Analogon der von Edinger als „centraler Schmerz" bezeichneten Erscheinung findet. Es ist auffallend, dass das letztere Symptom in meinem Falle vollständig gefehlt hat, da doch die starke Auseinanderzerrung der linken medialen Schleife im grössten Theil ihres Verlaufes durch die Brückengegend, sowie ihre Infiltration mit Geschwulstmasse und die an vielen Fasern nachweisbare Degeneration die günstigsten Bedingungen für Reizerscheinungen setzen musste. Unter welchen Umständen dieselben bei Affectionen der sensiblen Bahn auftreten oder fehlen, bleibt jedenfalls vor der Hand noch ganz im Dunkeln. Verständlich ist dagegen, dass die Sensibilität der gekrenzten Körperseite nicht aufgehoben, sondern nur vermindert war, weil eben nicht eine vollständige Unterbrechung der Schleifenbahn, sondern nur eine starke Verdrängung und theilweise Degeneration derselben stattgefunden hatte. Aus dem verhältnissmässig späteren Eintritt der Hypästhesie, die dann längere Zeit auf die obere Extremität beschränkt blieb nnd erst im letzten Krankheitsstadium auf die untere Extremität überging, ist zu schliessen, dass die Schleifenfasern zunächst nur durch die wachsende Geschwulstinfiltration auseinander gedrängt wurden und erst später einer theilweisen Degeneration verfielen. Ess zeigt sich an diesem Beispiel wieder, dass die Wirkung der Tumoren auf die Function der betroffenen Theile des Nervensystems eine andere ist, als die der hämorrhagischen und Erweichungsherde, und dass sowohl bei der klinischen Diagnose, wie bei den physiologischen Schlussfolgerungen dieser Verschiedenheit Rechnung getragen werden muss.

Dass vorübergehend auch Sensibilitätsabnahme in der linken unteren Extremität beobachtet wurde, ist aus der Betheiligung der in die linken Hinterstrangkerne übergehenden Kreuzungsfasern von der rechten medialen Schleife her zu erklären. Auch hier würde man nach dem anatomischen Befund eher eine vollständigere und anhaltende Sensibilitätsstörung erschlossen haben, muss also rückwärts wieder den Schluss ziehen, dass trotz starker Auseinanderzerrung und theilweiser Degeneration der Fasern doch ihre Leitungsfähigkeit nur vorübergehend beeinträchtigt wurde.

3. Ataxie wurde in meinem Falle während des ganzen Krank-

wohl angenommen werden, dass keine rollständige Unterbrechung der Sehleifenbahn, sondern nur eine sebr starke Compression und Degeneration vorgelegen hat. 
heitsverlaufes vermisst, obwohl im Hinblick auf die früheren Veröffentlichungen ähnlicher Fälle immer wieder nach diesem Symptom gefabndet wurde. Die einzige motorische Störung in den Extremitäten war eine mässige Abnahme der groben Kraft, die sich gleichzeitig mit der erwähnten Sensibilitätsabnahme einstellte und die wohl von der von der Geschwulst ausgehenden Compression der linken Pyramide abzuleiten ist. Eine Störungder Coordination der Bewegungen konnte dagegen nicht festgestellt werden. Wie aus der erwähnten Zusammenstellung von Moeli und Marinesko hervorgeht, wurde Ataxie theils mit theils ohne Sensibilitätsstörung bisher in solchen rällen von Brückenerkrankung gefunden, in welchen ein bestimmter (von den Autoren in ein Schema eingezeichneter) Abschnitt der Formati o re ticularis erkrankt war. Dieser Abschnitt fällt in meinem Falle, wie ein Vergleich der Figuren ergiebt, jedenfalls in das Bereich des Tumors; der letztere hätte also, wenn die Bedeutung jener Stelle in der That die vorausgesetzte ist, das Symptom der Hemiataxie hervorrufen müssen. Auch hier ist aber der Einwand berechtigt, dass der Tumor, soweit er nicht direct die nervöse Substanz vernichtet, sondern sie nur auseinanderdrängt und einen Theil der Fasern zur Degeneration bringt, nicht als gleichwerthig mit einem scharf abgegrenzten Erweichungs- oder Blutungsherd angesehen werden kann.

Wenn also in meinem Falle sowohl eine erhebliche Läsion der Schleife wie eine solche der Formatio reticularis und trotzdem keine Ataxie bestanden hat, so kann daraus nur der Schluss gezogen werden, dass die in anderen Fällen beobachtete Ataxie von der Zerstörung bestimmter, in die angegebenen Regionen fallender, aber bis jetzt noch nicht sicher zu bezeichnender Faserzüge abhängt und dass gerade diese Faserzüge in meinem Falle keine vollständige Unterbrechung erfahren haben.

Das Gleiche gilt für die Erkrankung der Oblongata, in welcher ebenfalls Querschnittstheile an der Läsion betheiligt waren, von deren Zerstörung man nach Analogie anderer Fälle das Symptom der Ataxie hätte erwarten müssen. Es ist dies die Region derSchleifenkreuzung. Erst in jüngster Zeit wieder ist durch Reinhold*) ein Fall von Herderkrankung in der Oblongata veröffentlicht worden, in welchem in Folge von Thrombose der rechten Arteria vertebralis der grösste Theil der rechten Seite der Oblongata zerstört war. Es hatte eine vollständige Lähmung und Sensibilitätsabnahme in der linken Körper-

*) Beiträge z. Pathol. der acuten Erweichungen des Pons a. der Oblongata. Deutsche Zeitschr. f. Nervenheilkunde. 1894. Bd. 5. Heft 4 und 5. 
hälfte bestanden, zugleich Ataxie der rechten oberen Extremität, welche letztere der Autor als bedingt durch die Mitbetheiligung der aus den rechten Hinterstrangskernen kommenden Schleifenfasern ansieht. Diese Fasern waren in meinem Falle unzweifelhaft auf der linken Seite betheiligt, indem sie durch Geschwulstinfiltration auseinandergedrängt und theilweise degenerirt waren. Wenn trotzdem die Ataxie auch auf der Seite der Läsion gefehlt hat, so ist zu schliessen, dass diese bei langsamer Compression der Fasern nicht einzutreten braucht, während andererseits ihr acuter Eintritt bei einer plötzlichen, rasch zum Tode führenden Zerstörung wie in dem Reinhold'schen Falle freilich auch noch nicht beweist, dass hier ein dauerndes Ausfallssymptom vorliegt. Es bleibt wünschenswerth, dass zur Aufhellung dieser Verhältnisse - namentlich auch was die Bèziehung der Ataxie zu den neuerdings besonders wieder von Goldscheider urgirten Muskelsinnstörungen betrifft - möglichst chronische Fälle mit constanten Symptomen und scharf umschriebenen Herden untersucht und veröffentlicht werden.

4. Die Dysphagie bildete in meinem Falle ein sehr prägnantes Symptom, das zunächst anfallsweise als vollständige Schluckunfähigkeit, dann danernd als erhebliche Beeinträchtigung des Schluckactes auftrat. Der anatomische Befund ergab eine Betheiligung aller beim Schluckacte betheiligten Nerven: des Facialis, des motorischen Trigeminus und des Vagus. Da, wie bereits ausgeführt wurde, der Tumor wahrscheinlich in der Gegend des Facialisursprungs und cerebralwärts hiervon seinen Ausgang genommen hatte, so ist es berechtigt, die ersten Anfälle von Dysphagie auf Facialis und Trigeminus zu beziehen und eine spätere Betheiligung des Vagus an den dauernden Schluckstörungen anzunehmen. Jedenfalls war der motorische Vaguskern (Nucleus ambiguus) nicht zerstört, sondern nur von Geschwulstmasse infiltrirt, während der Facialis- und der motorische Trigeminuskern vollständig zu Grunde gegangen waren. Sonstige auf den Vagus zu beziehende Erscheinungen - Störungen der Respirations- und Herzthätigkeit - wurden auffallender Weise vermisst, obwohl die Degeneration des sensiblen Vaguskerns links eine sehr erhebliche war und das solitäre (Respirations-) Bündel z war noch nachweisbar war, aber stark plattgedrückt und erheblich degenerirt erschien. $O b$ die schliesslich, nach zweijähriger Krankheitsdauer eingetretene tödtliche Pneumonie als Vaguspneumonie aufzufassen ist, muss dahingestellt bleiben.

5. Gehörstörungen wurden bei meiner Kranken nicht beobachtet. Ich mass aber bemerken, dass nur in der ersten Zeit der 
Beobachtung genauere Gehörprüfungen vorgenommen wurden, während in der späteren Zeit leider keine eingehendere Untersuchung in dieser Richtung mehr stattfand. Es kann also nur festgestellt werden, dass keine erhebliche Beeinträchtigung des Hörvermögens vorgelegen hat, während eine geringe Abnahme desselben möglicherweise der Beobachtung entgangen ist.

Wie sich aus der anatomischen Beschreibung ergiebt, hatte von demjenigen Theile des Acusticusursprungs, aus welchem der Nervus vestibuli hervorgeht, der dorsolaterale Kern tiefgreifende Veränderungen erfahren, während der Deiters'sche Kern ziemlich gut erhalten war: Der Ursprungskern des eigentlichen Hörnerven, des Nv. cochleae, hatte dagegen durch die Geschwulst nur eine starke seitliche Verschiebung erfahren, zeigte sich aber im Uebrigen, ebenso wie der Nerv selbst von ganz normaler Beschaffenheit.

Anders verhielt es sich freilich mit den centralen Forsetzungen dieses Nerven, die wir nach Flechsig, Held und Kölliker zunăchst vorwiegend in den Fasern des Corpus trapezoides und in den Striae acusticae zu suchen haben, weiterhin in der gekrenzten lateralen Schleife. Bekanntlich hat vor Kurzem Weinland*) unter Hinweis auf diesen anatomischen Zusammenhang das Vorkommen von Gehörstïrungen in seinem und in anderen Fällen von Vierhügelerkrankung auf eine Unterbrechung dieser Bahn zurückgeführt. In meinem Falle war nun ein Theil der linksseitigen Striae acusticae degenerirt, auch das Corpus trapezoides fiel noch zum Theil in die Gegend der Geschwulstinfiltration; ausserdem bestand aber in der linken lateralen Schleife, d. h. in dem Ursprungsgebiet des rechten Nerv. cochleae erhebliche Faserdegeneration. Man hätte also eine doppeltseitige Abnahme des Hörvermögens erwarten müssen. Möglicherweise war dieselbe, wie gesagt, in geringem Grade vorhanden; erheblich kann sie aber keinesfalls gewesen sein, da sie sonst gerade bei der gleichzeitigen Betheiligung beider Gehörnervenbahnen sicher aufgefallen wäre. Auch hier wird wohl an die Besonderheit der Geschwulstwirkung gedacht werden müssen, die trotz weitgehender Infiltration doch keine vollständige. Zerstörung der Bahnen zu Stande gebracht hat.

6. Schliesslich mögen noch einige Bemerkungen über die Natưr der Geschwulst Platz finden. Dass dieselbe als ein reines Gliom

*) Ueber einen Tumor der Vierbügelgegend und über die Beziehungen der hinteren Vierhügel za Gehörsstörungen (aus Prof. Flechsig's Laboratorium). Im vorigen Hefte dieses Archivs. 
bezeichnet werden muss, unterliegt nach dem Ergebnisse der mikroskopischen Untersuchung keinem $\mathrm{Z}$ weifel, wie mir auch Herr Professor 0. Israel, welcher die Freundlichkeit hatte, meine Präparate durchzusehen, bestätigt hat. Die Geschwulst bestand im Wesentlichen aus zahlreichen meist etwas länglichen Kernen, die mit denen der Neuroglia an Grösse und Färbbarkeit übereinstimmten und zwischen denen reichlich körnig amorphes $Z$ wischengewebe sich vorfand. Inmitten der Geschwulst, und zwar am stärksten in der Gegend ihrer grössten Ausdehnung fanden sich sebr zahlreiche stark erweiterte und prall gefüllte Gefässe mit ziemlich dünner Wand, die stellenweise ein so starkes Netz bildeten, dass sie wie Teleangiektasien erschienen. Nirgends waren aber an denselben Wandveränderungen oder Infiltrationen mit Rundzellen zu bemerken und nur ganz vereinzelt fanden sich minimale Blutaustritte. Ebenso erwiesen sich die grösseren Gefässe innerhalb und in der Umgebung der Geschwulst als durchaus normal. Nirgends waren Bilder zu finden, wie man sie bei syphilitischen und bei arteriosklerotischen Erkrankungen so zahlreich findet.

Von besonderem Interesse war das Verhalten der Geschwalst zu dem nervösen Gewebe. Ueberall zeigte sich weit über die makroskopisch als Grenze erscheinenden Ränder hinausgehend eine weit zerstreute Kerninfiltration des Nachbargewebes, durch welche die Nervenfasern auseinandergedrängt wurden und zunächst ein bei Weige r t färbung hervortretendes B las s e $r$ werden des Nervenmarks, sodann klumpige Gerinnungen und Abbröckelungen desselben erfuhren, während an den stärker afficirten Stellen anch Quellung der Axencylinder und Unterbrechung derselben eintrat. Nirgends war dagegen die bei myelitischen und Erweichungsherden so constante Reaction der nächsten Umgebung in Form von Körnchenzellen zu finden. Dieselben fehlten sowohl in den Gefässwänden wie im Zwischengewebe vollständig.

Während so ein Uebergreifen der Geschwulst weit über ihre scheinbaren Grenzen hinans constatirt werden konnte, war anderer. seits bis weit in ihr Inneres hinein das Vorkommen von Resten nervöser Substanz zu verfolgen. Vielfach fanden sich namentlich in der Gegend der Nervenkerne einzelne grosse offenbar gequollene $Z$ ellen, die durch ihre scharf begrenzten Kerne und Kernkörperchen und durch die abgestumpften Fortsätze sich als Ganglienzellen erwiesen. Noch viel zahlreicher waren namentlich an den Präparaten mit Pal carminfärbung die Reste des Nervenmarks nachuaweisen. Selbst in den centralsten Theilen der Ge- 
schwulst konnte man, wie Fig. 4 Taf. XIII. zeigt, fast in jedem Gesichtsfeld noch einzelne Klumpen von Nervenmark auffinden und je mehr man sich der Peripherie näherte, um so zahlreicher wurden die auf grössere Strecken verfolgbaren, allerdings vielfach veränderten Stücke markhaltiger Nervenfasern. Ich glaube nicht, dass es berechtigt wäre, nach diesen Befunden die Geschwulst als ein "Neurogliom" im Sinne von Klebs zu bezeichnen; denn überall zeigt doch das Nervengewebe nur die Erscheinungen der Rückbildung, nirgends die einer activen Betbeiligung an dem Process der Neubildung. Wohl aber ist dieses lange Erhaltenbleiben von Theilen der nervösen Elemente für die klinische Beurtheilung solcher Geschwulstformen von Bedeutung, indem sich ergiebt, dass auch in den von der Neubildung ganz durchsetzten Theilen doch immer noch erhebliche Mengen von möglicherweise leitungsfähigen Nervenfasern übrig bleiben. Würden wir ein ähnlich sicheres Verfahren zam Nachweis nackter Axencylinder besitzen, wie es zum Nachweis der Markhüllen zur Verfügang steht, so würden wahrscheinlich in noch viel grösserer Ausdehnung leitungsfähige $\mathrm{Fa}$ sern in solchen infiltrirten Gliomen aufgefunden werden können. Jedenfalls ermöglicht aber auch schon der vorliegende Befund das Verständniss solcher Fälle, in welchen, wie in dem seiner Zeit von Ladame*) beschriebenen, scheinbar die ganze Brückengegend durch eine Guschwulst ersetzt, die Nervenfasern derselben bis auf eine liniendicke Schicht an der Oberfäche geschwunden, und doch nur unerhebliche Störungen der Motilität und Sensibilität eingetreten waren. Würden damals schon die neneren Färbemethoden zur Verfügung gestanden haben, so hätten sich wohl zweifellos auch im Innern der Geschwulst zahlreiche Reste von Nervenfasern nachweisen lassen.

\section{Erklärung der Abbildungen (Tafel XIII.).}

Fig. 1. Tumor unter dem Boden des vierten Ventrikels, vorwiegend die linke Seite einnehmend. Abbildung in natürlicher Grösse nach dem bereits etwas erhärteten Präparat.

Fig. 2. Sohnitt aus der G'egend des Acusticus- und Facialisursprungs. Färbung nach $\mathrm{Pal}$ mit Carminnachfärbung. Linke Seite des Präparats grösstentheils ausgefüllt durch Gliommasse, welche hier in gelappter Form mit einer dorsalen und einer ventralen Einkerbung erscheint. Ventralwärts reicht der Tumor bis in die Gegend der Olive, welche letztere noch starke Infiltra-

*) Symptomat. und Diagnóstik der Hirngeschwülste. 1865. S. $123 \mathrm{ff}$. 
tion mit Gliomkernen zeigt. Schleife links blasser gefärbt als rechts, zeigt namentlich in ihrem dorsalen Abschnitt Kerninfiltration. Linke Pyramide in die Breite gezogen und vergrössert. Hinteres Längsbündel (A) rechts blasser und kleiner als normal, links kaum wahrnehmbar, erst bei stärkerer Vergrösserung in seinen Resten erkennbar.

A. Die in Fig. 3 bei stärkerer Vergrösserung gezeichnete Stelle.

B. Die in Fig. 4 bei stärkerer Vergrösserung gezeichnete Stelle.

Str. ao. Nach der Rapho zu verlaufende Bestandtheile der Striao acusticae.

F. asc. Faserzug, welcher die durch die Infiltration nach aussen gedrängte aufsteigende Wurzel des rechten $\mathrm{N}$. facialis enthält.

N. a. v. d. Rechter ventraler Acusticuskern; an dessen medialer Seite austretende Fasern des N. cochleae.

N. ac. dors. Rechter dorsolateraler (Deiters'scher) Acusticuskern.

N. a.. .s. Linker ventraler Acusticuskern mit Fasern des N. cochleae.

Fig. 3. Die Stelle A aus Fig. 2 bei stärkerer Vergrösserung. Gegend der hinteren Längsbündel, von welchen das rechte in gut gefärbten und regelmässig gestellten Faserquerschnitten erkennbar ist, während das linke nur in vereinzelten unregelmässigen Querschnitten erscheint, darunter einzeln $\theta$ vergrösserte Ringe und Bröckel pon Nervenmark. Die von rechts her in die Raphe eintretenden Fasern aus den Striae acusticae ziemlich gut erhalten, die von links her kommenden blass und unregelmässig gestellt, stellenweise wie abgebrochen.

Fig. 4. Die Stelle B ans Fig. 2 bei stärkerer Vergrösserung. Partie aus der Mitte des Tumors. Das Gewebe besteht ans Kernen mit amorpher Zwischensubstanz. Zahlreiche dünnwandige Gefässe, in deren Umgebung sich stärkere Kernanbänfung findet. Mitten in dem Geschwulstgewebe noch an einzelnen Stellen Reste von Nervenmark in Form von Kugeln und von länglich unregelmässigen Bröckeln. 


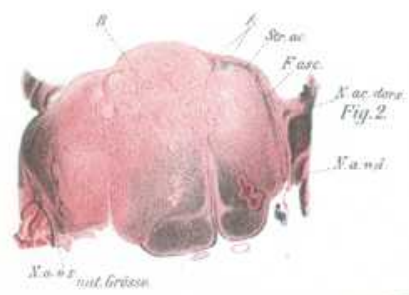

Frig. 4. $(B$ andirly 2$)$

Fing. 3.
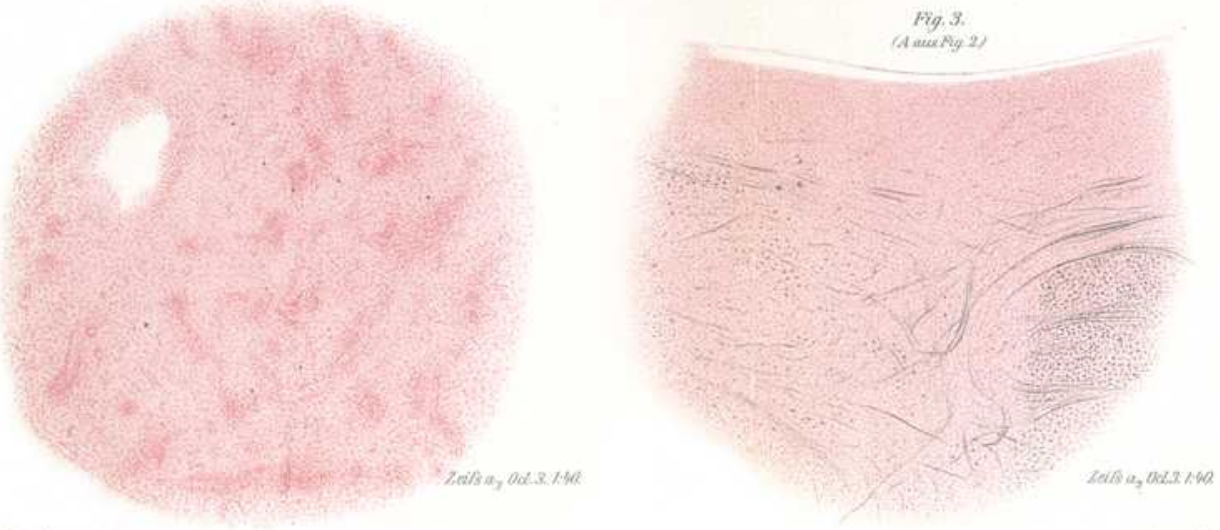